\title{
Neoclassical Ion Transport in the Edge of Axially-Symmetric Arbitrary Cross-Section Tokamak with Plasma Subsonic Toroidal Flows
}

\author{
J.H.F. Severo, V.S. Tsypin, I.C. Nascimento, R.M.O. Galvão, \\ M. Tendler ${ }^{1}$, and A. N. Fagundes \\ Institute of Physics, University of São Paulo, \\ Rua do Matão, Travessa R, 187, 05508-900 São Paulo, Brasil \\ 1 The Alfvén Laboratory, EURATOM-Nuclear Fusion Research, \\ Royal Institute of Technology, 10044 Stockholm, Sweden
}

Received on 26 June, 2001

\begin{abstract}
General metrics of large aspect ratio tokamaks is used in the paper. General expressions for the neoclassical poloidal plasma rotation $V_{i}^{\theta}$ and radial ion heat flux $\Gamma_{T i}$ are obtained. Their dependence on the squared Mach number $\alpha=U_{\zeta i}^{2} / c_{s}^{2}$ is analyzed (here $U_{\zeta i}$ is the ion toroidal velocity and $c_{s}$ is the sound velocity, respectively). Some interesting peculiarities of this dependence are emphasized.
\end{abstract}

\section{Introduction}

The theoretical and experimental study of the neoclassical ion transport in edge tokamak plasmas are nowadays of renewed interest. ${ }^{1-4}$ In particular, these studies are important in the so-called H-regimes in large toroidal facilities, characterized by sharp gradients in the radial profiles of macroscopic plasma quantities in the region of the transport barriers. ${ }^{1,2}$ Plasmas of small tokamaks, which play an important role in investigating different physical phenomena in fusion research, are mainly in L-regime. Nevertheless, some problems regarding the neoclassical ion transport in these machines are not yet well understood and additional efforts are needed. ${ }^{3,4}$ One of these problems is addressed in this paper, namely the neoclassical ion transport in the edge of an axisymmetric plasma column of arbitrary crosssection and with subsonic toroidal flows. These flows can be induced by parallel neutral beam injection or by radio frequency waves.

\section{Starting equations}

The main neoclassical quantities observed experimentally are the ion radial heat flux and the ion poloidal velocity. ${ }^{1,2}$ Until now there is no satisfactory theoretical explanation for the damping rate $\tau_{\zeta}$ of toroidal rotation, which is usually supposed to be approximately equal to the energy confinement time $\tau_{E}, \tau_{\zeta} \approx \tau_{E}{ }^{5}$

We follow Refs. 6 and 7, where the magnetic surface averaged ion radial heat flux for collisional plasmas was first obtained. We use the toroidal coordinates $r, \theta, \zeta$, where $r$ is the magnetic surface label, and $\theta$ and $\zeta$ are poloidal and toroidal angles, respectively, assume axial symmetry, i.e., $\partial / \partial \zeta=0$, large aspect ratio, and smooth profiles of the macroscopic plasma quantities. The magnetic surface average

$$
\langle\ldots\rangle=\int_{0}^{2 \pi}(\ldots) \sqrt{g} d \theta / \int_{0}^{2 \pi} \sqrt{g} d \theta
$$

of the radial contravariant component $q_{i}^{r}$ of the ion heat flux (see Ref. [8]),

leads to

$$
q_{i}^{r}=-\frac{2 p_{i} \nu_{i}}{M_{i} \omega_{c i}^{2}}\left(g^{11} \frac{\partial T_{i}}{\partial r}+g^{12} \frac{\partial \widetilde{T}_{i}}{\partial \theta}\right)-\frac{5}{2} \frac{p_{i} g_{33} B^{\zeta}}{M_{i} \omega_{c i} B \sqrt{g}} \frac{\partial \widetilde{T}_{i}}{\partial \theta}
$$

$$
\Gamma_{T i}=\left\langle q_{i}^{r}\right\rangle=-\frac{2}{M_{i}}\left\langle\frac{p_{i} \nu_{i} g^{11}}{\omega_{c i}^{2}} \frac{\partial T_{i}}{\partial r}\right\rangle-\frac{5}{2} \frac{c}{e_{i}}\left\langle g_{33} B^{\zeta}\right\rangle \int_{0}^{2 \pi} d \theta \frac{p_{i}}{B^{2}} \frac{\partial \widetilde{T}_{i}}{\partial \theta} / \int_{0}^{2 \pi} \sqrt{g} d \theta
$$


Here, $T_{i}$ is the ion temperature, $\widetilde{T}_{i}=T_{i}-\left\langle T_{i}\right\rangle$ is the oscillatory part (perturbation) of the ion temperature, $p_{i}=n T_{i}, n=\langle n\rangle+\widetilde{n}$ is the plasma density and $\widetilde{n}$ is the oscillatory part of it, $\omega_{c i}=e_{i} B / c M_{i}$ is the ion cyclotron frequency, $B$ and $B^{\zeta}$ are the magnitude and the $\zeta$-contravariant component of the magnetic field $\mathbf{B}$, respectively, and $g^{11}$ and $g^{12}$ are the contravariant components of the metric tensor $\widehat{\mathbf{g}}$, whose determinant is denoted $g$.

In obtaining Eq. (3), we used also the expressions for the contravariant components of the magnetic filed

$$
\mathbf{B}=\left\{0 ; \quad \chi^{\prime} / 2 \pi \sqrt{g} ; \quad \phi^{\prime} / 2 \pi \sqrt{g}\right\}
$$

where $\chi$ and $\phi$ are poloidal and toroidal magnetic filed fluxes, respectively, and

$$
\partial\left(g_{33} / \sqrt{g}\right) / \partial \theta=0 .
$$

The last equation follows from the condition $j^{r} \approx$ 0 , where $j^{r}$ is the $r$-contravariant component of the plasma current j. The inequality $\widetilde{A}=A-\langle A\rangle \ll\langle A\rangle$ was also employed, where $A$ stands for the plasma macroscopic quantities.

We see from Eq. (3) that, in order to find $\Gamma_{T i}$, we need to calculate $\widetilde{n}$ and $\widetilde{T}_{i}$. The equation for $\widetilde{T}_{i}$ follows from the ion heat transport equation ${ }^{8}$

$$
-T_{i} V_{i}{ }^{\theta} \frac{\partial \tilde{n}}{\partial \theta}+\frac{B^{\theta}}{B} \frac{\partial q_{i \|}}{\partial \theta}+\nabla \cdot \mathbf{q}_{i \perp}+\frac{3 M_{e} n \nu_{e}}{M_{i}} \tilde{T}_{i}=0
$$

where $V_{i}^{\theta}$ is the $\theta$-contravariant component of the ion velocity $\mathbf{V}_{i}$, and

$$
q_{i \|}=-3.91 \frac{n T_{i} B^{\theta}}{M_{i} \nu_{i} B} \frac{\partial T_{i}}{\partial \theta}, \quad \mathbf{q}_{i \perp}=\frac{5}{2} \frac{c n T_{i}}{e_{i} B^{2}}\left[\mathbf{B} \times \nabla T_{i}\right]
$$

Perturbations of the electron temperature are neglected in Eq. (6) (see explanations after Eq. (18)). To find $\tilde{n}$ one can employ the oscillatory part of the parallel component of the plasma one-fluid momentum equation

$$
-\frac{3}{2} \pi_{\|} \frac{\partial}{\partial \theta} \ln B+\frac{\partial\left(p+\pi_{\|}\right)}{\partial \theta}+M_{i} n \frac{\mathbf{B}}{B^{\theta}} \cdot \frac{d_{i} \mathbf{V}_{i}}{d t}=0
$$

where $p=p_{i}+p_{e}=n\left(T_{i}+T_{e}\right)$ is the plasma pressure and $\pi_{\|}$is the parallel ion viscosity, ${ }^{9,10}$ and

$$
\frac{d_{i} \mathbf{V}_{i}}{d t}=\frac{\partial \mathbf{V}_{i}}{\partial t}+\left(\mathbf{V}_{i} \cdot \nabla\right) \mathbf{V}_{i}
$$

Here we used also the well-known approximate expression (see, e.g., Ref. 3)

$$
\boldsymbol{\nabla} \cdot \boldsymbol{\pi}=\frac{3}{2}\left\{[\mathbf{h} \boldsymbol{\nabla} \cdot \mathbf{h}+(\mathbf{h} \cdot \boldsymbol{\nabla}) \mathbf{h}] \pi_{\|}+\mathbf{h}(\mathbf{h} \cdot \boldsymbol{\nabla}) \pi_{\|}-\frac{1}{3} \boldsymbol{\nabla} \pi_{\|}\right\},
$$

where $\pi$ is the ion viscosity tensor. The velocity component $V_{i}^{\theta}$ can be found from the poloidal average of the parallel component of the momentum equation (8)

$$
\int_{0}^{2 \pi} d \theta\left(M_{i} n \frac{\mathbf{B}}{B^{\theta}} \cdot \frac{d_{i} \mathbf{V}_{i}}{d t}-\frac{3}{2} \pi_{\|} \frac{\partial \ln B}{\partial \theta}\right)=0 .
$$

The parallel ion viscosity $\pi_{\|}$, entering Eqs. (8) and (9), is defined by ${ }^{3,4,9,10}$

$$
\pi_{\|}=-\frac{2}{3} \frac{p_{i}}{\nu_{i}}(0.96 \beta-0.59 \gamma)
$$

where

$$
\begin{aligned}
& \beta=3\left\{-V_{i}^{\theta} \frac{\partial}{\partial \theta} \ln \left(\sqrt{g} n^{2 / 3} B\right)+\frac{B^{\theta 2}}{B^{2}} V_{i}^{\theta} \frac{\partial g_{22}}{\partial \theta}+V_{T i} \frac{\partial}{\partial \theta} \ln \frac{B}{n}\right\}, \\
& \gamma=-3\left\{0.34 V_{i}^{\theta} \frac{\partial}{\partial \theta} \ln n+V_{T i}\left(1.36 \frac{\partial}{\partial \theta} \ln B-0.84 \frac{\partial}{\partial \theta} \ln n\right)\right\},
\end{aligned}
$$

and

$$
V_{T i}=\frac{c}{e_{i}\left\langle B^{2}\right\rangle}\left\langle\left[\mathbf{B} \times \nabla T_{i}\right]^{\theta}\right\rangle=\frac{c}{e_{i}} \frac{g_{33}}{\sqrt{g}\left\langle B^{2}\right\rangle}\left\langle B^{\zeta} \frac{\partial T_{i}}{\partial r}\right\rangle .
$$


Thus, we have Eq. (3) to determine the radial heat flux $\Gamma_{T i}$, and Eq. (9) to find the $\theta$-contravariant component of the plasma velocity $V_{i}^{\theta}$. To calculate them we should solve Eqs. (6), (8), and (9).

\section{Solution of the perturbed equations}

We find from Eq. (8)

$$
\frac{\mathbf{B}}{B^{\theta}} \cdot \frac{d_{i} \mathbf{V}_{i}}{d t}=-\frac{1}{2} U_{\zeta i}^{2} \frac{\partial \ln g_{33}}{\partial \theta}
$$

and, consequently,

$$
\frac{\partial\left(\widetilde{p}+\pi_{\|}\right)}{\partial \theta}=\alpha \frac{\langle p\rangle}{2} \frac{\partial \ln g_{33}}{\partial \theta},
$$

where $\alpha=M_{i} U_{\zeta i}^{2} /\left(T_{i}+T_{e}\right)$ is the squared Mach number, $\widetilde{p}=\widetilde{n}\left(T_{i}+T_{e}\right)+n \widetilde{T}_{i}$. To obtain Eq. (14), we used the covariant differentiation rules

$$
\nabla_{i} V^{k}=\frac{\partial V^{k}}{\partial x^{i}}+\Gamma_{i m}^{k} V^{m}
$$

and imposed that the metric tensor components $g_{12}=$ $g_{21}$ are periodical functions of the angle $\theta$.

Using Eqs. (6) and (7), we obtain the second order differential equation for the perturbed ion temperature $\widetilde{T}_{i}$,

$$
\frac{\partial^{2} \widetilde{T}_{i}}{\partial \theta^{2}}-2.17 b \sqrt{\frac{M_{e}}{M_{i}}} \widetilde{T}_{i}=f(\theta)
$$

where

$$
f(\theta)=\frac{0.51 b T_{i}}{\nu_{i}}\left[\left(\frac{5}{2} V_{T i}-V_{i}^{\theta}\right) \frac{\partial \ln n}{\partial \theta}-5 V_{T i} \frac{\partial \ln B}{\partial \theta}\right]
$$

$b=B^{2} / \lambda_{i}^{2} B^{\theta 2}$ is the collisionality parameter, and $\lambda_{i}=\sqrt{2 T_{i} / M_{i}} / \nu_{i}$ is the ion mean free path. The order of the parameter $B^{2} / B^{\theta 2}$ for large aspect ratio tokamaks is approximately $q^{2} R^{2}$, where $q$ is the safety factor and $R$ is the torus major radius. Thus we approximately have $b=q^{2} R^{2} / \lambda_{i}^{2}$. For the collisional plasma the parameter $b>1$. We consider the range $1<b \lesssim \sqrt{M_{i} / M_{e}}$ in this paper. In this case one can omit perturbations of the electron temperature in Eqs. (6) and (17). For the range $1<b \lesssim M_{i} / M_{e}$, these perturbations should be taken into account (see, e.g., Ref. $3)$.

As far as the function $f(\theta)$ and, consequently, $\widetilde{T}_{i}$ are periodical in $\theta$ and moreover, proportional to $\sin \theta$ for the circular cross-section tokamak ${ }^{3}$ and to $\sin \theta$ and $\sin 2 \theta$ for elliptical cross-section tokamak, ${ }^{4}$ the solution of Eq. (17) has the form

$$
\widetilde{T}_{i}=\sum_{s=1}^{\infty} \widetilde{T}_{i s} \sin s \theta
$$

where

$$
\widetilde{T}_{i s}=-\int_{0}^{2 \pi} \frac{f(\theta) \sin s \theta d \theta}{\left[\pi\left(s^{2}+2.17 b \sqrt{M_{e} / M_{i}}\right)\right]} .
$$

Comparison of Eq. (15) with Eqs. (17)-(20) shows that, to zero approximation, one can use

$$
\frac{\partial \ln n}{\partial \theta} \approx \frac{\alpha}{2} \frac{\partial \ln g_{33}}{\partial \theta} .
$$

This expression can be substituted into Eqs. (3), (11), (12) and (18). The surface averaged parallel component of the momentum equation (9), taking into account Eq.

(8), can be transformed into the form

$$
\int_{0}^{2 \pi} d \theta\left\{\widetilde{\pi}_{\|}\left(\frac{3}{2} \frac{\partial \ln B}{\partial \theta}-\frac{\alpha}{2} \frac{\partial \ln g_{33}}{\partial \theta}\right)-\langle n\rangle \widetilde{T}_{i} \frac{\alpha}{2} \frac{\partial \ln g_{33}}{\partial \theta}\right\}=0 .
$$


Using also the identity

$$
B^{2}=g_{22} B^{\theta 2}+g_{33} B^{\zeta 2},
$$

we get

$$
\frac{\partial \ln B}{\partial \theta} \approx \frac{1}{2}\left(\frac{1}{q^{2} R^{2}} \frac{\partial g_{22}}{\partial \theta}-\frac{\partial \ln g_{33}}{\partial \theta}\right) .
$$

Thus we can express every perturbed value via the os- cillatory parts of the metric components $g_{22}$ and $g_{33}$.

\section{Ion fluxes}

Let us simplify expressions for perturbed values using Eqs. (21) and (24). The Fourier component $\widetilde{T}_{i s}$ of the perturbed ion temperature $\widetilde{T}_{i}$ has the form

$$
\widetilde{T}_{i s}=-\frac{1.3 b T_{i}}{\nu_{i} d_{s}(b)}\left\{\left[V_{T i}\left(1+\frac{\alpha}{2}\right)+\frac{\alpha}{5} V_{i}^{\theta}\right] \int_{0}^{2 \pi} \sin s \theta \frac{\partial \ln g_{33}}{\partial \theta} d \theta-\frac{V_{T i}}{q^{2} R^{2}} \int_{0}^{2 \pi} \sin s \theta \frac{\partial g_{22}}{\partial \theta} d \theta\right\},
$$

where $d_{s}(b)=s^{2}+2.17 b \sqrt{M_{e} / M_{i}}$. The parallel viscosity $\pi_{\|}$, Eqs. (10)-(12), can be expressed in the form

$$
\begin{aligned}
\pi_{\|}= & \frac{0.96 p_{i}}{\nu_{i}}\left\{V_{i}^{\theta}\left[(1+0.46 \alpha) \frac{\partial \ln g_{33}}{\partial \theta}-\frac{1}{q^{2} R^{2}} \frac{\partial g_{22}}{\partial \theta}\right]+\right. \\
& \left.+1.83 V_{T i}\left[(1+0.83 \alpha) \frac{\partial \ln g_{33}}{\partial \theta}-\frac{1}{q^{2} R^{2}} \frac{\partial g_{22}}{\partial \theta}\right]\right\} .
\end{aligned}
$$

Equation (22) can be transformed into

$$
\int_{0}^{2 \pi} d \theta \pi_{\|}\left[\left(\frac{3}{2}+\alpha\right) \frac{\partial \ln g_{33}}{\partial \theta}-\frac{3}{2 q^{2} R^{2}} \frac{\partial g_{22}}{\partial \theta}\right]+\alpha\langle n\rangle \sum_{s=1}^{\infty} \widetilde{T}_{i s} \int_{0}^{2 \pi} d \theta \sin s \theta \frac{\partial \ln g_{33}}{\partial \theta}=0 .
$$

Substituting Eqs. (25) and (26) into Eq. (27), one finds the poloidal velocity $V_{i}^{\theta}$ (to be compared with Ref. 12),

$$
V_{i}^{\theta}=-1.83 V_{T i} f_{2}(\alpha, b, A, D) / f_{1}(\alpha, b, A, D),
$$

where

$$
\begin{gathered}
f_{1}(\alpha, b, A, D)=\left(1+\frac{2}{3} \alpha\right)(1+0.46 \alpha) A_{33}+A_{22}-(2+1.13 \alpha) A_{23}+0.36 \alpha^{2} b D_{33}, \\
f_{2}(\alpha, b, A, D)=\left(1+\frac{2}{3} \alpha\right)(1+0.83 \alpha) A_{33}+A_{22}- \\
-(2+1.5 \alpha) A_{23}-0.48 \alpha b\left[\left(1+\frac{\alpha}{2}\right) D_{33}-D_{23}\right] \\
A_{33}=\int_{0}^{2 \pi} d \theta\left(\frac{\partial \ln g_{33}}{\partial \theta}\right)^{2} \\
A_{22}=\frac{1}{q^{4} R^{4}} \int_{0}^{2 \pi} d \theta\left(\frac{\partial g_{22}}{\partial \theta}\right)^{2} \\
A_{23}=\frac{1}{q^{2} R^{2}} \int_{0}^{2 \pi} d \theta \frac{\partial \ln g_{33}}{\partial \theta} \frac{\partial g_{22}}{\partial \theta}, \\
D_{33}=\sum_{s=1}^{\infty} \frac{1}{d_{s}(b)}\left(\int_{0}^{2 \pi} d \theta \sin s \theta \frac{\partial \ln g_{33}}{\partial \theta}\right)^{2} \\
\frac{1}{q^{2} R^{2}} \sum_{s=1}^{\infty} \frac{1}{d_{s}(b)} \int_{0}^{2 \pi} d \theta \sin s \theta \frac{\partial \ln g_{33}}{\partial \theta} \int_{0}^{2 \pi} d \theta \sin s \theta \frac{\partial g_{22}}{\partial \theta} .
\end{gathered}
$$

Equation (3) for the surface averaged ion heat flux can be rewritten as follows

$$
\Gamma_{T i}=\left\langle q_{i}^{r}\right\rangle=-\frac{2\left\langle p_{i} \nu_{i}\right\rangle\left\langle g^{11}\right\rangle}{M_{i}\left\langle\omega_{c i}^{2}\right\rangle}\left\langle\frac{\partial T_{i}}{\partial r}\right\rangle-\frac{5}{2} \frac{c}{e_{i}}\left\langle\frac{p_{i}}{B^{2}}\right\rangle\left\langle g_{33} B^{\zeta}\right\rangle \int_{0}^{2 \pi} d \theta \widetilde{T}_{i} \frac{\partial}{\partial \theta} \ln \left(\frac{B^{2}}{n}\right) / \int_{0}^{2 \pi} \sqrt{g} d \theta .
$$


Substitution of Eqs. (19), (25), (21), and (24) into Eq. (36) results in

where

$$
\begin{aligned}
& \Gamma_{T i}=\left\langle q_{i}^{r}\right\rangle=-\frac{2 p_{i} \nu_{i}}{M_{i} \omega_{c i}^{2}} \frac{\partial T_{i}}{\partial r}\left\langle g^{11}\right\rangle\left\{1+0.8 q^{2}\left\langle g_{33}^{2}\right\rangle\left[\left(1+\frac{\alpha}{2}\right)^{2} D_{33}-(2+\alpha) D_{23}+D_{22}-\right.\right. \\
& \left.\left.-0.37 \alpha\left(\left(1+\frac{\alpha}{2}\right) D_{33}-D_{23}\right) f_{2}(\alpha, b, A, D) / f_{1}(\alpha, b, A, D)\right] /\left(\left\langle g^{11}\right\rangle\langle\sqrt{g}\rangle \int_{0}^{2 \pi} \sqrt{g} d \theta\right)\right\},
\end{aligned}
$$

$$
D_{22}=\frac{1}{q^{4} R^{4}} \sum_{s=1}^{\infty} \frac{1}{d_{s}(b)}\left(\int_{0}^{2 \pi} d \theta \sin s \theta \frac{\partial g_{22}}{\partial \theta}\right)^{2}
$$

\section{Estimates}

Let us analyze the expressions for the ion poloidal velocity Eq. (28) and the ion heat flux Eq. (37) in a general case. When the squared Mach number $\alpha$ vanishes, $\alpha=0$, one obtains from Eq. (28)

$$
V_{i}^{\theta}=-1.83 V_{T i}
$$

which agrees with results of Refs. 11, 3, and 2 . Equations (28) and (39) also confirm the Hazeltine theorem, ${ }^{13}$ which says that the so-called residual plasma poloidal rotation in tokamaks depends only on the ion temperature gradient and not on gradients of other macroscopic plasma parameters. Estimates show that the parameters $A_{23}$ and $D_{23}$ are negative, $\left(A_{23}<0\right.$ and $\left.D_{23}<0\right)$. Hence, the parameter $f_{1}(\alpha, b, A, D)$ is positive, $f_{1}(\alpha, b, A, D)>0$, i.e., the denominator in Eqs. (28) and (37) is positive and has no roots as a function of $\alpha$. A remarkable property of the poloidal velocity $V_{i}^{\theta}$ is the change of sign at a value $\alpha_{0}$ of the parameter $\alpha$. This results from the fact of taking into account inertial forces in the starting equations. Assuming that the poloidal velocity changes sign at $\alpha<1$ (see in detail Ref. 11), we find from Eq. (30)

$$
\alpha_{0} \approx 2.1\left(A_{33}+A_{22}-2 A_{23}\right) /\left[b\left(D_{33}-D_{23}\right)\right] .
$$

From the approximate equation,

$$
V_{i}^{\theta} \approx 0.88 V_{T i} \frac{\alpha b\left(D_{33}-D_{23}\right)}{\left(A_{33}+A_{22}-2 A_{23}+0.36 \alpha^{2} b D_{33}\right)},
$$

one finds the critical quantity $\alpha_{k}$,

$$
\alpha_{k} \approx 1.67 \sqrt{\frac{A_{33}+A_{22}-2 A_{23}}{b D_{33}}},
$$

corresponding to the maximum of the poloidal velocity $V_{i(\max )}^{\theta}$,

$$
V_{i(\max )}^{\theta} \approx 0.73 V_{T i} \frac{\sqrt{b}\left(D_{33}-D_{23}\right)}{\sqrt{D_{33}\left(A_{33}+A_{22}-2 A_{23}\right)}} .
$$

For $\alpha>\alpha_{k}$, the poloidal velocity $V_{i}^{\theta}$ decreases slowly with the growth of $\alpha$.

Analysis of Eq. (37) shows that the magnetic surface averaged radial ion heat flux $\Gamma_{T i}$ is an increasing function of the parameter $\alpha$. The factors that characterize the non-circularity of the plasma cross-section, such as ellipticity, triangularity, reduce the role of neoclassical effects in $\Gamma_{T i}$ for all values of the parameter $\alpha$. These results coincide with the previous studies of this problem, fulfilled for elliptical and circular cross-section tokamaks, ${ }^{3,4}$ and we demonstrate them here.

\section{Elliptical tokamak}

In the case of the elliptical tokamak we find from Eqs. (28)

$$
U_{i \theta}=G_{u}(\alpha, b) U_{T i}
$$

where $U_{i \theta}=\rho V_{i}^{\theta}, U_{T i}=\left(1 / M_{i} \omega_{c i}\right) \partial T_{i} / \partial \rho, \rho=\sqrt{l_{1} l_{2}}$, $l_{1}$ and $l_{2}$ are the semiminor and semimajor axes of a tokamak cross-section, respectively,

$$
\begin{gathered}
G_{u}(\alpha, b)=-\frac{f_{2}(\alpha, b)}{f_{1}(\alpha, b)}, \\
f_{1}(\alpha, b)=d(b)\left(1+\frac{2}{3} \alpha\right)(1+0.19 \alpha)+0.18 \alpha^{2} b, \\
f_{2}(\alpha, b)=d(b)\left(1+\frac{2}{3} \alpha\right)(1.83+1.52 \alpha)-0.88 \alpha b\left(1+\frac{\alpha}{2}\right),
\end{gathered}
$$


$d(b)=1+2.2 b \sqrt{M_{e} / M_{i}}$. Obtaining Eq. (44), the parameter

$$
A=\frac{l_{1}}{R q}\left(\frac{l_{2}^{2}}{l_{1}^{2}}-1\right)
$$

was assumed to be small, $A \ll 1$.

Using Eq. (37), we derive the magnetic surface average of the radial ion heat flux in the Shafranov form, ${ }^{6}$

where

$$
\Gamma_{T i}=-\frac{2 n T_{i} \nu_{i}}{M_{i} \omega_{c i}^{2}} \frac{\partial T_{i}}{\partial \rho} \frac{\left(l_{1}^{2}+l_{2}^{2}\right)}{2 l_{1} l_{2}}\left[1+3.2 q^{2} \frac{l_{1}^{2}}{\left(l_{1}^{2}+l_{2}^{2}\right)} G_{T}(\alpha, b)\right]
$$

$$
\begin{gathered}
G_{T}(\alpha, b)=\left(1+\frac{\alpha}{2}\right)\left(1+\frac{2}{3} \alpha\right) \frac{f_{3}(\alpha)}{f_{1}(\alpha, b)} \\
f_{3}(\alpha)=\left(1+\frac{\alpha}{2}\right)(1+0.19 \alpha)+\frac{\alpha}{5}(1.83+1.52 \alpha) .
\end{gathered}
$$

Equations (44) and (49) coincides with the proper equations of Ref. 4. As far as the coefficient $l_{1}^{2} /\left(l_{1}^{2}+l_{2}^{2}\right)$ in Eq. (49) is less than $1, l_{1}^{2} /\left(l_{1}^{2}+l_{2}^{2}\right)<1$, the role of neoclassical effects in the radial ion heat flux decreases in noncircular cross-section tokamaks in comparison with circular cross-section ones.

The quantities $G_{u}(\alpha, b)$ and $G_{T}(\alpha, b)$ are plotted in Figs. 1 and 2, respectively. One can see that function $G_{u}(\alpha, b)$ (Fig. 1$)$ changes sign at $\alpha_{0} \approx 2 d(b) / b$. For the collisional parameter $b \sim \sqrt{M_{i} / M_{e}}$, the quantity $\alpha$ is equal to $\alpha_{0} \approx 0.1$. The maximum of function $G_{u}(\alpha, b)$ is achieved when $b_{m} \approx 50, \alpha_{m} \approx 1$, and is $G_{u}\left(\alpha_{m}, b_{m}\right) \approx 3$. It follows from Fig. 2 that the neoclassical contribution in the radial ion heat flux, as a function of $\alpha$, is dropping with increasing $b$.

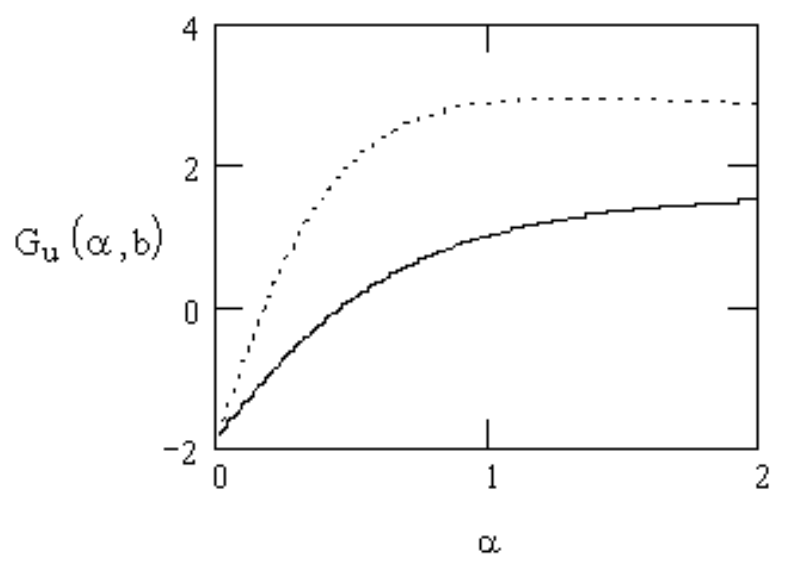

Figure 1. The dependence of the function $G_{u}(\alpha, b)$ on $\alpha$ for different magnitudes of the parameter $b$ : $b_{1}=10$ (solid curve) and $b_{2}=50$ (dashed curve).

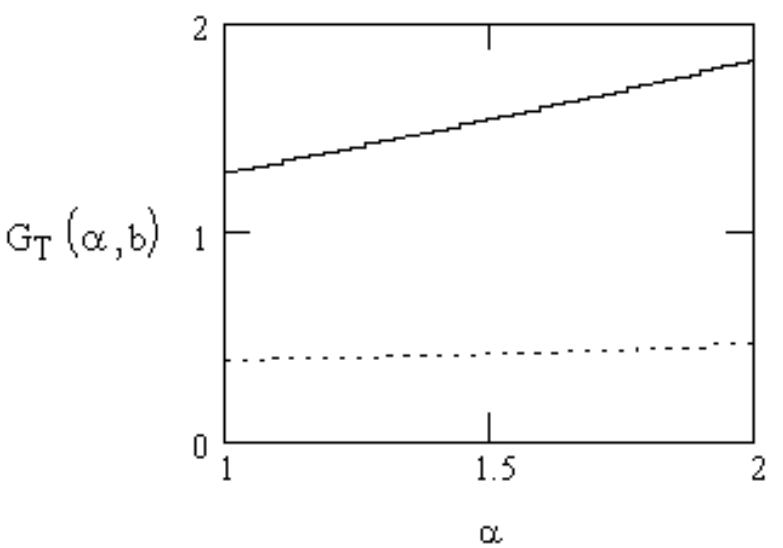

Figure 2. The dependence of the function $G_{T}(\alpha, b)$ on $\alpha$ for different magnitudes of the parameter $b: b_{1}=10$ (solid curve) and $b_{2}=50$ (dashed curve).

\section{Conclusions}

General expressions for neoclassical poloidal plasma rotation $V_{i}^{\theta}$ and radial ion heat flux $\Gamma_{T i}$ for an axiallysymmetric arbitrary cross-section tokamak edge with plasma subsonic toroidal flows are obtained in the present paper. Their dependence on the squared Mach number $\alpha$ is analyzed. It is shown that there is a remarkable property of the poloidal velocity $V_{i}^{\theta}$ to change sign at a value $\alpha=\alpha_{0}$, which results from taking into account inertial forces in the starting equations. There also exists a critical value of $\alpha, \alpha_{k}$, which corresponds to the maximum of the poloidal velocity $V_{i(\max )}^{\theta}$. For $\alpha>\alpha_{k}$, the poloidal velocity $V_{i}^{\theta}$ is a decreasing function of $\alpha$. Analysis of the magnetic surface averaged radial ion heat flux $\Gamma_{T i}$ shows that this flux is an increasing function of the parameter $\alpha$. The noncircular cross-section of a tokamak decreases the role of neo- 
classical effects in $\Gamma_{T i}$ for any value of the parameter $\alpha$. These results confirm previous studies of this problem.

\section{Acknowledgments}

This work was supported by the Research Support Foundation of the State of São Paulo (FAPESP), National Council of Scientific and Technological Development $(\mathrm{CNPq})$, and Excellence Research Programs (PRONEX) RMOG 50/70 grant from the Ministry of Science and Technology, Brazil.

\section{References}

[1] A. Rogister, Phys. Rev. Lett. 81, 3663 (1998).

[2] H. A. Claassen, H. Gerhauser, A. Rogister, and C. Yarim, Phys. Plasmas 7, 3699 (2000).

[3] V. S. Tsypin, D. Kh. Morozov, J. J. E. Herrera, J. J. Martinell, M. Tendler, I. F. Potapenko, A. S. de Assis, and C. A. de Azevedo. Plasma Phys. Control. Fusion 39, 1681 (1997).
[4] V. S. Tsypin, C. A. de Azevedo, and A. S. de Assis, Physics Letters A. 219, 282 (1996).

[5] K. Brau, M. Bitter, R. J. Goldston, D. Manos, K. McGuire, and S. Suckewer, Nucl. Fusion 23, 1643 (1983).

[6] V. D. Shafranov, Sov. Atom. Energy 19, 1008 (1965).

[7] V. D. Shafranov, J. W. Connor, and C. J. Watson, Sov. J. Plasma Phys. 2, 99 (1976).

[8] S. I. Braginskii, in Reviews of Plasma Physics, edited by M. A. Leontovich (Consultants Bureau, New York, 1965), Vol. 1, p. 205.

[9] A. B. Mikhailovskii and V. S. Tsypin, Plasma Physics 13, 785 (1971).

[10] A. B. Mikhailovskii and V. S. Tsypin, Beiträge Plasma Physik 24, 335 (1984).

[11] A. B. Mikhailovskii and V. S. Tsypin, Sov. Phys. JETP 56, 75 (1982).

[12] B. N. Kuvshinov, Sov. J. Plasma Phys. 16, 227 (1990).

[13] R. D. Hazeltine, Phys. Fluids 17, 961 (1974). 